\title{
THE POSITIVE EFFECT OF SWIMMING ON THE MOVEMENT SYSTEM
}

\section{Sobir Usmonovich Nazarov}

Associate Professor, Department of "Sports Management", Institute Of Retraining And Advanced Training Of Specialists In Physical Culture And Sports, Uzbekistan

\section{ABSTRACT}

The article discusses the positive effects of swimming on the human body. In particular, the effects of

butterfly, brass, crawl methods on the musculoskeletal system, increased resistance to diseases of acute respiratory viral infections, improvement of the functional state of the body were discussed.

KEYWORDS:- Musculoskeletal system, butterfly, brass, crawl, acute respiratory viral infections (ARVI), endurance, muscle elasticity, psychoemotional state.

\section{INTRODUCTION}

The development of physical culture and sports in our country has become one of the priorities of state policy. The reason is that only a harmoniously developed generation with a physically healthy, spiritually mature and independent mind can build a great state of the future. Therefore, the resolutions and decrees adopted by the head of our state, the resolutions of the Cabinet of Ministers identify problems related to the development of physical culture and sports and their solutions.

The process of renewal and development of all spheres is being actively carried out in the country, and great attention is paid to the science of physical culture and sports.

Nowadays, in the context of the growth of scientific and technical tables, the issues of adaptation of the organism to the environment are cross-cutting. In particular, these ideas apply to the young organism. By the same period, the acquisition and enrichment of vital skills and movements is observed.

Swimming is a form of physical education. Its benefits in strengthening the body and making the body healthier are invaluable. Swimming is one of the most beneficial types of exercise.

Swimming is an effective prophylactic against acute respiratory viral infections ARVI (influenza, parainfluenza, adenovirus disease, reovirus disease, respiratory syncytial infection and rhinovirus disease), as well as a sport that closely helps in maintaining the health of people of different ages.

The benefits of swimming have been covered in many sources, including the beneficial effects of this sport on human health.

It is known that the fetus is in contact with the aquatic environment from the time of development in the mother's womb. This is why newborns are not afraid of water treatments, 
bathing and diving. Thus, the aquatic environment is comfortable and natural for humans.

It is also one of the effective tools in strengthening the immune system. Many years of practical experience show that the incidence of acute respiratory viral infections (ARVI) in primary swimming training groups is higher than in training exercise groups. (Table 1)

\section{Table 1.}

\section{Percentage of total morbidity in groups.}

\begin{tabular}{|l|c|c|c|}
\hline \multicolumn{1}{|c|}{ Periods } & BT-1 & BT-3 & O'MG $^{\prime}$ 'M \\
\hline September 2017 & $25 \%$ & $18 \%$ & - \\
\hline December 2017 & $30 \%$ & $20 \%$ & - \\
\hline September 2018 & $20 \%$ & $17 \%$ & $12 \%$ \\
\hline December 2018 & $18 \%$ & $15 \%$ & $10 \%$ \\
\hline
\end{tabular}

Note:

BT-1 Primary Preparatory Group 1 year

BT-3- Primary Preparatory Group 3 years

O’MG-training exercise group

But as we have noted, the beneficial effects of water occur through regular participation in the swimming pool at least 2-3 times.

How does swimming affect our body?

There is a lot of information about the beneficial effects of this sport on our body and health. We outline the key points to consider.

Swimming in the pool for an hour two to three times a week makes us 80 percent more resistant to viruses and germs, even in the fall and during a flu epidemic. By mastering professional swimming techniques (dragging, breaststroke, butterfly stroke, etc.), we learn to breathe again and develop the lung workforce.

Because swimming has almost no restrictions, unlike other sports, it does not allow the body to receive high levels of physical and psychological loads, which can be practiced by people of different ages due to their health, swimming respiratory, circulatory, musculoskeletal systems, including: has a positive effect on the spine, joints, and is an excellent antidepressant.

According to a 2014 experiment by Russian scientists working with children with musculoskeletal disorders and children with high mobility, it was found that swimming takes a long time (1 year or more) and only through regular exercise to have a positive effect. is achieved.

Swimming is also one of the most effective means of combating overweight, as additional muscle groups are activated during swimming (it is known that an average of $600 \mathrm{kcal}$ of energy is expended during 45 minutes of swimming). Swimming can be considered as one of the popular sports that provides practical assistance to overweight children.

The reason for the abundance of breathing 
exercises in swimming exercises is that it has a beneficial effect on the development of the respiratory system, thereby leading to an increase in the vital capacity of the lungs and a decrease in respiratory rate.

All this leads to an improvement in the functional state of the body.

Interviews with sports school coaches revealed that coaches tend to include swimming in their curriculum. Such measures help trainers to solve various problems: for skiers, in particular - to increase endurance performance; for gymnasts to achieve joint, muscle elasticity and load on the spine; to players - to improve coordination of actions; to restore the psycho-emotional state of chess players and others. In addition, such measures shorten the recovery period of the athlete's body and increase the volume of special loads.

To determine the demand for this type of physical education and sports, when Russian scientists conducted a survey in 2014 among parents of pupils of MBUDO 1-DYUSh (BOSM) "LIGR" in Novosibirsk (368 participants), 40\% of students in various sports regularly go to the pool. showed participation. Swimming has become important among all segments of the population.

Indeed, the role of swimming in our lives is unique: it is one of the most beneficial types of physical activity, it has a positive effect on the human body in all respects, promotes health on a physical and mental level.

On the issue of interdependence of disciplines, the direction of physical education and physical culture education students should focus on the interdependence of swimming in the transition from the subject of anatomy to the basic movement system. Accordingly, today we want to talk about the role of water sports in the development of the musculoskeletal system in student athletes, the issues of its positive qualities.

The human body contains more than 600 muscles, more than 200 bones, which are connected by mutual joints and joints. The benefits of swimming are invaluable in strengthening the body and making it healthier. Because when a person swims in water, all his muscles move. Consequently, the human body develops in proportion as a whole. The joints of a child who is regularly engaged in swimming are mobile, strong, move in a moderate manner, the limbs and muscles are well developed. One of the main reasons for this is that the horizontal movement in water is somewhat different from the movements a person performs while standing.

While standing exercises have a one-way effect on a person's locomotor system, swimming exercises have a one-way effect on him. In humans, there are diseases of the spine, such as kyphosis, scaliosis, lordosis. These diseases are caused by young children sitting incorrectly at a desk, table, chair, not fully following the rules of various exercises when doing sports. This in turn leads to the incorrect formation of the base movement system. This is why swimming is so important in preventing such diseases. In the treatment of people with such diseases is recommended to engage in swimming. Of course, it will be necessary to take additional treatments at the same time as swimming.

There are several methods of swimming (dolphin, brass, crawl), the performance of which, of course, depends on the proper development of the musculoskeletal system in athletes.

If there are any defects in the musculoskeletal system, it will certainly make it difficult to perform swimming techniques correctly, reduce the effectiveness of the exercise, and require the athlete to perform additional movements, which 
will consume a lot of energy, muscle fatigue, heart palpitations and rapid fatigue.

Although the mobility of the arms in water sports seems to depend only on the position of the shoulder joints, in fact their free and harmonious movement depends on the position of the spine, more precisely on the flexibility of the thoracic and lumbar spine. That is, in butterfly swimmers, if the thoracic-shoulder portion of the spine is not sufficiently mobile, the arm will be forced to lift the upper body involuntarily when performing wrist training exercises. This in turn leads to the development of this part of the base locomotors system.

In breaststroke swimming, it is important that the pelvis, especially the knee joint, and the ankle joints move as freely as they want. Because in any of these joints, if the movements are a little bit limited, it becomes difficult to perform wide stretching movements with the legs.

If the mobility and flexibility of the cervical spine is weak, the crawl swimmer will be forced to lift his head up without turning to the side, as required for breathing. In sports, not only 5-10 $\mathrm{cm}$ of anger, but also 5-10 $\mathrm{mm}$ of extra movement is superfluous, as raising the head of a swimmer upwards further undermines the shape and harmony of the general movements. This, in turn, affects the body's excessive vibration, the general condition of the body, and disrupts the athlete's swimming process.

Athletes do not perform well when swimming with the legs, lying on their chests or backs with the crawl method, when the movement in the calf joints is slow. The reason is that when a person swims in this way, mainly the toes of the feet move the swimmer forward. Therefore, the structure of the paws and their position during swimming are of great importance.

With this in mind, we recommend that swimmers pay close attention to the movement of their joints, depending on their swimming style, and train them non-stop during training.

When swimming is practiced, the musculoskeletal system is well developed in athletes. It has a positive effect on the proper development of the musculoskeletal system, especially if you start to engage in water sports at an early age. Especially the back, chest, shoulder muscles are well developed. The leg muscles also develop properly.

It is known that muscles contract or lengthen depending on the content, continuity of impulses coming from the lower centers of the brain. Depending on this, the foot or hand makes a movement. Consequently, it does mechanical work and at the same time moves the human body, moving it forward.

The swimmer usually develops the method of his choice in a series of exercises, and in this regard he begins to understand the technique of swimming.

Only an athlete who is able to embody the swimming technique in his brain can achieve a lot. Because all actions are known to him from thread to needle. Now he has to make this habit, which he knows and perceive, by using the appropriate muscles. The quality of a swimmer's technique is determined by how well he or she is able to save energy and prepare for the event.

Proportionality in body structure is to some extent important in mastering swimming techniques. For example, average swimmers with well-developed long-legged weights may have slightly higher performance. This can be seen in the activities of world-renowned swimmers. In most cases, tall, arms and legs are relatively light. It is no coincidence that 14-17-year-old adolescent swimmers often have high performance due to light weight, although their muscle development is not sufficient.

That is why these aspects are taken into account 
when choosing a future swimmer. In an adolescent athlete, there may be various changes in the body as an adult. For this reason, anthropometric examination is carried out from time to time, but it should be noted that the height of the swimmer's height and arm-leg length and small body weight cannot be a guarantee of his success, but only serve as a basis for it. In order to achieve high results in sports, first of all, the functional capacity of the floating organism depends on the methods of movement, which are based on the skills and abilities acquired during training.

In the dolphin method, swimmers are usually of medium height, the body is long, the legs are relatively short, the muscles of the shoulders, torso, arms and legs are developed early, and the joints are mobile. Athletes swimming in the backstroke method have high shoulders, long arms and legs, well-developed shoulder and arm muscles, broad chest, and mobile joints.

In the brass method, most swimmers are of medium height, heavy weight, especially the pelvic floor muscles, as well as the welldeveloped thigh and leg muscles. It is also known that their legs are the strongest energetic. The mobility of their shoulder joints is relatively low, but their knee and leg claw joints are so mobile that this opportunity is very handy in the brass method, and so on.

Muscles of physical workers, regular physical education and sports have a large amount of reserves of substances such as ATF, KF, glycogen, which are a source of energy in the muscles. Therefore, such people do not get tired quickly when exercising, because the above substances are broken down and provide the energy needed for muscle work.

Most people recognize professional swimmers among strangers, paying attention to the shape that suits them and the unique confidence and strength they hold.
Why pay more attention to this particular sport?

First, swimming is a universal sport in which all muscles move with the same load.

Second, swimming is safe for our joints and spine. People who have problems with the locomotor system but don't want to give up movement find a great way to give their bodies the energy charge they need when swimming.

Third, swimming helps to strengthen the immune system and increase the body's protective function.

The popular SPA abbreviation in Latin, Sanis Pierre Aqua, means "health from the water," which means that cool, clean water not only cleanses when touched, but also heals and energizes the body and soul.

There are no age or gender restrictions for swimming. Both adults, children and the elderly can enjoy maximum opportunities for health and physical development from this sport. Swimming is equally beneficial to man in any situation. Regular exercise in the pool will help their bodies feel new and prepare for childbirth, which will be easier for pregnant mothers in the pool after special exercises.

\section{Conclusion}

From the above data, it can be seen that the normal development of the elements of the musculoskeletal system can be achieved in athletes through swimming. If the musculoskeletal system develops normally in the athlete, it will certainly lead to the proper and positive development of the respiratory system, blood circulation, digestion, nervous system, which are important systems of the body. Therefore, it is recommended that athletes engage in additional water sports, regardless of the type of sport. This, of course, leads to an increase in their physical qualities, the proper development of the musculoskeletal system. 
CURRENT RESEARCH JOURNAL OF PEDAGOGICS 2(10): 220-225

October 2021 DOI: https://doi.org/10.37547/pedagogics-crjp-02-10-40

ISSN 2767-3278

(C)2021 Master Journals

Crossref doi) 81 Google

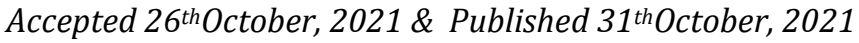

Proper development of the locomotor system in all sports closely helps the athlete to achieve high results.

In fact, proper swimming is another pride of many professional swimmers, as it allows air to be pumped directly from the lungs into the water during movement, endurance, developed chest and strong voice.

\section{REFERENCES}

1. Decree of the President of the Republic of Uzbekistan dated February 7, 2017 No 4947 "On the Action Strategy for further development of the Republic of Uzbekistan".

2. Resolution of the Cabinet of Ministers of the Republic of Uzbekistan 2018

3. Resolution No. 333 of May 3 "On measures to develop swimming sports."

4. A.A.Rixsieva, F.N.Nasriddinov, A.I.Rixsiev The physical condition of schoolchildren and athletic youth. UZGIFK, 1992

5. Bulgakova, N.J. Poznakomtes - swimming, first steps in sports / N.J. Bulgakova. - M .: 000 «Izdatelstvo AST», 2002

6. Geyger A.I., Pulatxujaeva M. Rules of swimming competitions.//Teaching manual. Toshkent 2015. 36.bet.

7. Zavadovskaya E.N., Ikramov F.T. Methods of developing special endurance of swimmers.//Method manual. Toshkent 2012. 31.bet.

8. Corbut V.M. SWIMMING (sports pedagogical improvement). $-\mathrm{T} \quad$. Lider Press, 2012, 142 p.

9. Krasnova G.M. Swimming history. A study guide for teachers, students, and MOF listeners. T., O'z.DJTI 2010

10. Sodiqov A.G. Schedule training sessions for young swimmers. $-\mathrm{T} \quad . . \quad$ «Science and technology», 2015

11. http://www.rsl.ru

12. http://aquatics.uz

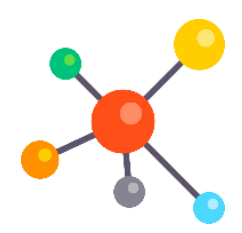

